\title{
Oxidation of hexafluoropropylene with molecular oxygen
}

\author{
Egbert Meissner, Agnieszka Wróblewska \\ Institute of Organic Chemical Technology, Szczecin University of Technology, ul. Pułaskiego 10, 70-322 Szczecin, Poland, \\ e-mail: Agnieszka.Wroblewska@ps.pl
}

\begin{abstract}
The results of the oxidation of hexafluoropropylene (HFP) to hexafluoropropylene oxide (HFPO) have been presented. The oxidation was carried out in an autoclave within the range of temperatures of $120-170^{\circ} \mathrm{C}$ and in the presence of an organic solvent (carbon tetrachloride). Molecular oxygen was used here as an oxidizing agent. The influence of: the molar ratio of HFP/O from 13.4 to 4.09:1, the process temperature, the addition of inert gas (nitrogen) from 4 to $18 \mathrm{~atm}$ and the periodical dosing of oxygen were investigated. The functions describing the process were: the conversion of HFP and the yield of HFPO in relation to HFP consumed. The presented process is very interesting owing to a wide application of HFPO.
\end{abstract}

Keywords: hexafluoropropylene, hexafluoropropylene oxide, epoxidation, oligomerization.

\section{INTRODUCTION}

The main product of hexafluoropropylene (HFP) oxidation is heksafluoropropylene oxide (HFPO). HFPO is a valuable intermediate for the synthesis by the oligomerization of perfluoroalifatic compounds with the structure of a linear polyether which is ended with a fluorocarbonyl group. This is the easiest method of receiving perfluoroalifatic compounds because it is characterized by a small number of stages from a raw material to a final product. Up to now no better oxidation method has been presented. Each of the so far used methods has got their advantages and disadvantages. There is still much work regarding the improvement of all the known methods as well as that of looking for the new ones.

The standard methods of receiving HFPO are based on the oxidation of HFP with hydrogen peroxide in the alkaline medium and at the temperatures below $-30^{\circ} \mathrm{C}^{\mathbf{1 , 2}}$ or with t-butyl hydroperoxide in the solvent with a molybdenum carbonyl catalyst, in the range of temperatures of 60 $-150^{\circ} \mathrm{C}$ and under the pressure of $20-70 \mathrm{at}^{3}$. The next method of receiving HFPO is based on the oxidation of HFP with sodium hypochlorite in the biphasic system and in the presence of the phase transfer catalyst ${ }^{4}$. A nonpressure method of the oxidation of HFP with oxygen and in the presence of active silica gel and at the temperature above $240^{\circ} \mathrm{C}$ has also been described ${ }^{5}$. The method of oxidation with oxygen and without a catalyst under the increased pressure in an organic solvent and at mild temperatures $\left(130-165^{\circ} \mathrm{C}\right)$ seems to be very interesting because it does not generate many by-products in comparison with the old methods ${ }^{6}$. It is also an ecologicallyfriendly method as it generates small amounts of wastes.

The oxidation of HFP is the process which is more complicated than for example, the oxidation of propylene, due to different chemical properties of HFP and HFPO. In contrast to the propylene oxide the chlorohydrine method cannot be used here. The receiving of oxide in that case is impossible owing to its unstable structure. The main problem here is caused by the decomposition of HFPO at temperatures above $165^{\circ} \mathrm{C}^{7}$ :
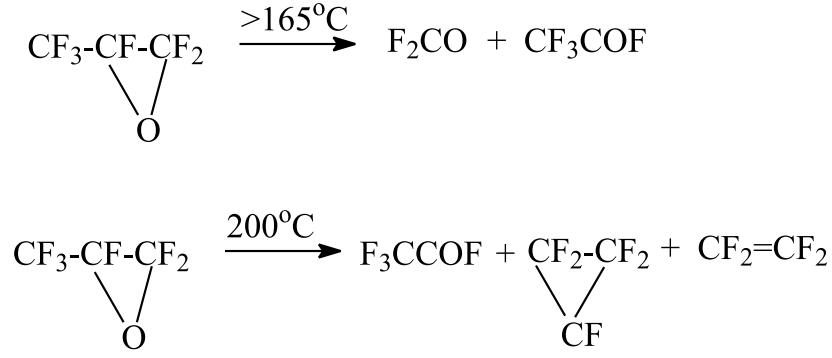

The aim of this work was to establish the optimal parameters of the oxidation HFP to HFPO with molecular oxygen. The influence of the following parameters was studied, that is: the molar ratio of HFP/O from 13.4 to 4.09:1, the process temperature from $120-170^{\circ} \mathrm{C}$, the addition of inert gas (nitrogen) from 4 to 18 at and the periodical dosing of oxygen. The main functions describing the process were as follows: the conversion of HFP and the yield of HFPO in relation to the HFP consumed.

\section{EXPERIMENTAL}

The oxidation of HFP was performed in a swinging autoclave of the $500 \mathrm{~cm}^{3}$ capacity, made of chromiumnickel-molybdenum steel made by Lampart (Hungary). The autoclave was heated with electricity and the regulation of the heat was performed manually by an autotransformer. The autoclave was equipped with: 2 needle valves, a thermometer pocket, a manometer and a safety valve. Into the dry autoclave, which was examined with the view to eliminating potential leak, the proper amount of the solvent (carbon tetrachloride) was poured. After closing the cover, the autoclave was cooled in the cryostat to the temperature $-50^{\circ} \mathrm{C}$ and the container with HFP (standing on the balance) was connected by a flexible hose, to one valve but the other valve was connected with the vacuum pump. After receiving the temperature $40^{\circ} \mathrm{C}$ in the autoclave, the air was eliminated and the vacuum pump was cut off. Later the proper amount of HFP was put into the autoclave. After filling up the autoclave with HFP it was taken out of the cryostat and left to receive the ambient temperature and after that, the autoclave was filled up with the precisely weighed out amount of oxygen using a special pressure feeder. After closing the oxygen valve, the autoclave was put into the rocking cradle and the heating and swaying was turned on. 
After some time the autoclave was taken out of the cradle and cooled to the ambient temperature and then unloaded by freezing the received gas products in the cryostat at $-60^{\circ} \mathrm{C}$. While the distillation of the products of the reaction was being performed, the gas samples were taken and then subjected to chromatographic analyses in order to perform the quantitative and qualitative examinations of the post-reaction mixture. The constant parameters of the oxidation process were as follows: the reaction time was $25 \mathrm{~min}$ and the amount of the solvent comprised (5.38 $\mathrm{CCl}_{4}: 1 \mathrm{HFP}$ ).

The isolation of the HFP and HFPO could not be performed directly from the post-reaction mixture because those compounds have close boiling points: $-29,4^{\circ} \mathrm{C}$ and $-27.4^{\circ} \mathrm{C}$, respectively. The isolation of HFPO from the post-reaction mixture was made by the photobromination of the products of the reaction. The apparatus for the bromination consisted of a four-neck-flask of the capacity of $1500 \mathrm{~cm}^{3}$, equipped with a thermometer and a dropping funnel. The dropping funnel was filled up with bromine and the dropping was regulated using the teflon valve. The speed of dosing of gas products was regulated by the rotameter which was situated in the front of the flask. The reaction flask was illuminated from the outside with a mercuric lamp. There was another reactor, on top of the flask, with a fluorescent lamp being built in the middle. The product of the reaction of the bromination with the unreacted bromine were collected at the bottom of the flask but the gases leaving the reactors went into the reflux condenser where the products of the bromination and the unreacted bromine were condensed and came back through the trap to the previously condensed liquid products. The products not reacting with $\mathrm{Br}_{2}$ were sent into the scrubber in which the unreacted gases from the bromination process were neutralized. The HFPO, which was received in that way, was directed to the freezer situated in the cryostat. This isolated HFPO was examined using the gas chromatograph in order to establish its purity. Chromatographic analyses were performed using the Chrom-5 apparatus. The conditions of the analysis were as follows: two series of connected capillary columns, the first one was $27.5 \mathrm{~m}$ long and $0.53 \mathrm{~mm}$ in diameter and the other one was $30 \mathrm{~m}$ long and $0.32 \mathrm{~mm}$ in diameter; the stationary phase - RT-QPLOT; carrier gas - nitrogen $\left(4 \mathrm{~cm}^{3} / \mathrm{min}\right)$; the temperature of the thermostat $70^{\circ} \mathrm{C}$; the FID detector; the size of the sample was $10 \mu 1$.

\section{RESULTS}

The studies were conducted in order to establish the optimal conditions of the oxidation of HFP to HFPO. These conditions should help to decrease the amount of by-products (mainly carbonyl fluoride and fluoride trifluoroacetate acid) in the post-reaction mixture. The influence of the molar ratio of $\mathrm{HFP} / \mathrm{O}$ on the conversion of HFP and the yield of the HFPO in relation to HFP consumed is presented in Fig. 1. With the increase of the molar of HFP/O a decrease of the conversion of HFP as well as the yield of the HFPO from 33 to $9 \%$ vol. and from 30 to $6 \%$ vol. have been observed. We could not use the molar ratio $\mathrm{HFP} / \mathrm{O}$ below 4 because at this molar ratio the reaction proceeds explosively.

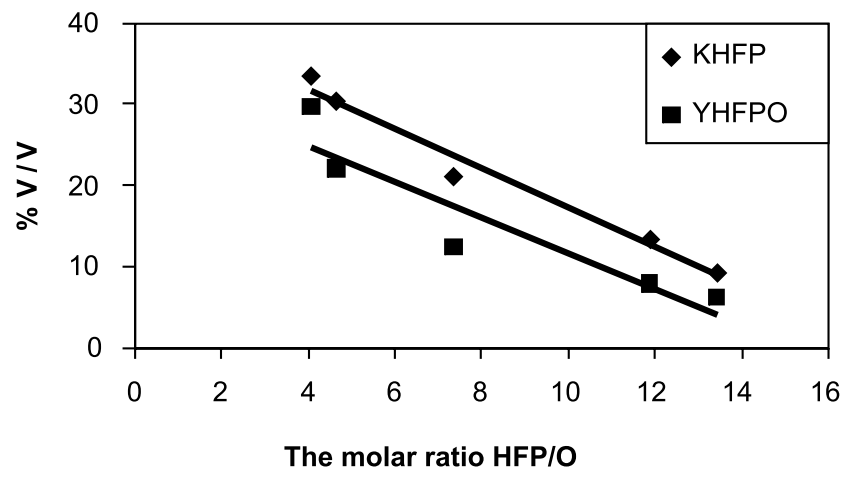

Figure 1. The influence of the molar ratio $\mathrm{HFP} / \mathrm{O}$ on the conversion of HFP (KHFP) and the yield of the HFPO (YHFPO)

The process temperature was the next studied parameter - Fig. 2. It results from the course of the curves that the conversion of HFP increases in the range of temperatures $120-175^{\circ} \mathrm{C}$ from $10 \%$ vol. do $35 \%$ vol. but the yield of HFPO increases in the range of temperature 120 $-155^{\circ} \mathrm{C}$ (from $8 \%$ vol. do $23 \%$ vol.) and next decreases insignificantly in the range of temperature $155-175^{\circ} \mathrm{C}$ (from $23 \%$ vol. to $21 \%$ vol.). These changes in the yield of HFPO are caused by the decomposition of HFPO to carbonyl fluoride and fluoride trifluoroacetate acid.

Fig. 3 presents the influence of the addition of the inert gas on the oxidation process of HFP - in the experiment nitrogen was used as inert gas. From the course of the curves it results that the influence of nitrogen presence on the oxidation process is positive in the case of the HFP conversion as well as the yield of HFPO. It is connected with the following phenomenon: the higher pressure the better solubility of gases in the liquid.

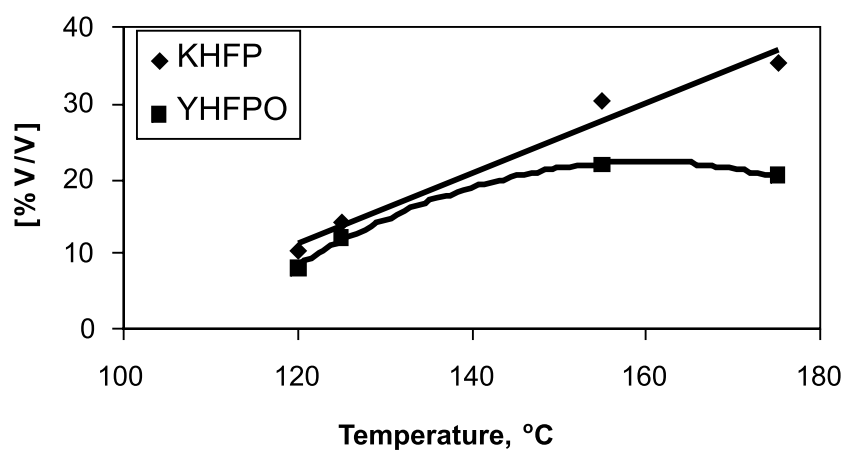

Figure 2. The influence of the temperature on the conversion of HFP (KHFP) and the yield of the HFPO (YHFPO)

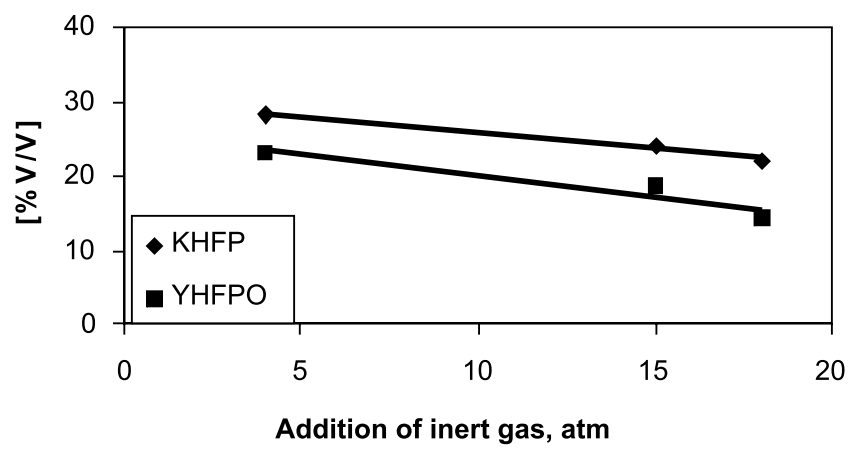

Figure 3. The influence of the inert gas addition on the conversion of HFP (KHFP) and the yield of the HFPO (YHFPO) 
Apart from the above parameters the influence of the periodically dosing of oxygen was also examined. The results of the studies showed that the periodic dosing of oxygen (two and three times) causes an insignificant increase in the yield of HFPO. It could be connected with the non-precise dosing of oxygen.

\section{CONCLUSION}

The performed studies showed that the main product of the oxidation of HFP in an organic solvent and under the increased pressure is HFPO. The by-products were: carbonyl fluoride and fluoride trifluoroacetate acid. They were formed in small amounts. The most important parameter among the examined ones was the molar ratio of $\mathrm{HFP} / \mathrm{O}$ as well as the temperature. The addition of nitrogen as an inert gas demonstrates minor influence. The addition of the inert gas leads to the increase in the solubility of the reagents in the liquid phase. The solvent was carbon tetrachloride, there is no doubt that any other solvent could be applied in which the solubility of both olefine and oxygen would be better, for example organic fluorocompounds. The periodic dosing of oxygen was not precise. Currently we are designing a precise oxygen feeder, working under the higher pressure so in the future we will study this parameter again. In the studies a very important parameter, that is the time of the reaction, was completely omitted. In the tests a constant time of 25 minutes was taken and it may be too short at low temperatures but too long at high temperatures. So this parameter should also be studied in the future in the extended scope.

Summing up we have to state that the process of the oxidation of HFP using oxygen, among many other methods, seems to be the method worth recommending due to the fact that oxygen is the cheapest oxidizing agent. The proposed method is not a complicated one despite using higher pressure $(25-35$ at).

\section{LITERATURE CITED}

(1) Patent RFN 2026677, 1970.

(2) Eleuterio H. S.: Polymerization of perfluoro epoxides,

J. Macromol. Sci. Chem., 1972, A6, 1027.

(3) Patent US 2614129, 1952.

(4) Patent US 4902810, 1990.

(5) Patent US 3775439, 1972.

(6) Patent RFN 1240516, 1972.

(7) Hill J. T.: Octafluoroisobutylene oxide derivatives, J. Fluorine Chem., 1977, 9(2), 97. 